\title{
On the Extension of a Star Based Parallel Interconnection Network Topology
}

\begin{abstract}
Due to architectural innovations design of parallel interconnection network topology is always an area of research. The Star graph is very popular among existing topologies. The present attempts modify star graphs with the application of extension technique. The different topological parameters of the proposed topology are derived. The comparison of topological and performance parameters is carried out to establish the suitability of the proposed network as a better candidate for large scale parallel system.
\end{abstract}

[ Nibedita Adhikari]
Keywords-interconnection network, fault tolerance, parameters, reliability, Isoefficieney

\section{Introduction}

The interconnection networks are the backbone of any parallel computing system [1]. Major parallel interconnection networks (IN) are Hypercube, Crossed cube, Star graphs [2, 3, 4, and 5]. With architectural innovations the basic structures are modified. The modification techniques resulted in various new topologies like Folded hypercube [6], Extended hypercue [7], Extended Varietal hypercube (EVH) [8] and Metacube [9] etc.

The EVH is a recursive and fault-tolerant interconnection topology called extended varietal hypercube $(\mathrm{EVH})$ is introduced in [8]. It has reduced diameter, average distance and constant degree of nodes. The Extended crossed cube (ECQ) [10] possess better diameter than the Crossed cube and Hypercube. Also the mean distance between vertices is shorter than that of the hypercube.

The Star graph is a popular alternative to the Hypercube with reduced diameter and constant degree of nodes [5]. The $\mathrm{n}$-Star is a node-symmetric and edgesymmetric graph consisting of $n$ ! number of nodes and $n$ ! $(n-1) / 2$ number of edges. In spite of its admitted superiority, the $n$-Star has a major disadvantage that is; it grows to its next higher dimension by a very large value. For example, the 4-Star contains 24 nodes where as, for a 5-Star network 5! = 120 nodes are needed. The considerable gap between the two consecutive sizes of the $\mathrm{n}$-Star is considered to be a major drawback and therefore needs further attention for its improvement. For this reason a new alternative called Extended Star (ES) was proposed [11].

Dr Nibedita Adhikari

Biju Patnaik University of Technology, Rourkela, Odisha, India
The details like construction, topological parameters and routing algorithms are discussed. The current paper is an extension of the same to discuss about the performance parameters like cost effectiveness, terminal reliability, packing density and scalability etc., in order to find the suitability of ES network as a candidate for large scale parallel system. The scalability of a parallel system is a measure of its capacity to increase speedup in proportion to the number of Processing Elements (PE) [12]. It is measured in terms of the Isoefficiency function which is the ratio of speedup to the total number of processors in the system. Packing density is the total number of PEs in the system.

The section two presents briefly the network and its parameters. The performance parameters are discussed in section 3. The Section 4 discusses the results of comparison of ES network against the parent networks. Section 5 concludes the paper.

\section{Extended Star Topology}

The Extended star is a variation of the n-Star graph [5]. The details of the proposed ES architecture are described in earlier work [11]. For convenience brief explanation about structure and basic topological parameters are presented below.

\section{A. Construction}

Basically the Extended star (ES) is a hierarchical topology with basic module as n-Star graph and one network controller which helps the processing elements to solely concentrate on computational work. An ES consisting of an n-Star and one $\mathrm{NC}$ forms the basic module ES(n,1) and is shown in Fig.1.

\section{B. Addressing}

The address-labeling scheme is a basic step to construct the multicomputer system as it helps in deciding the shortest diameter. The basic building block for an ES is an n-star. containing $\mathrm{n}$ ! number of nodes. The node addresses are permutations of $1,2,3, \ldots$. For example in 3 -Star there are six nodes and the node addresses are 123, $213,312,132,231,321$ respectively. Currently the node addresses are given in decimal notations that is $0,1,2,3$, 4 ... (n!-1) for simplicity. So in $\operatorname{ES}(3,1)$, the $\mathrm{NC}$ will have address 0 . The six children nodes will have node address 00, 01, 02, 03, 04, 05 as shown in Fig 1(a). The address of NC precedes the address of the node. The $\operatorname{ES}(3,2)$ is shown in Fig. 1(b) with addresses of the NC's only. 


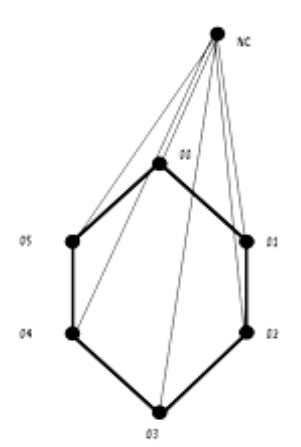

(a)

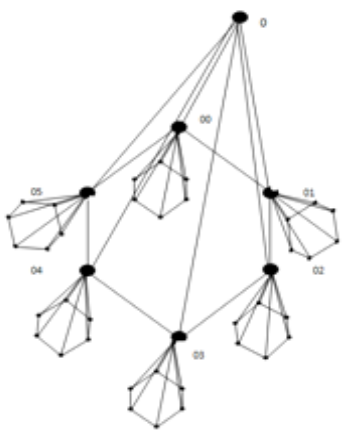

(b)

\section{Parallel Paths}

In the basic module ES(3,1), there are three parallel paths between any two nodes as shown in Fig. 2. For example, the parallel paths between 01 and 03 are given by

a) $01-02-03$

b) $01-00-05-04-03$

c) $01-0-03$

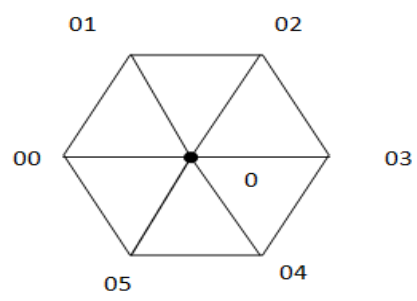

Figure 2. Parallel Paths in ES(3,1)

\section{Spanning Tree}

The procedure for generating the spanning tree of ES network is as follows:

a) The NC becomes the root.

b) Two farthest nodes become the children of the root.

c) Next the remaining odd/even nodes are placed as grand children of even/odd node.

The spanning tree for the basic module is shown in Fig. 3(a). For next higher level the spanning tree is constructed by appending the spanning tree of the basic module at all the nodes as shown in Fig. 3 (b).

\section{E. Topological Parameters of ES}

In this section the various topological parameters of the Extended Star graph are discussed briefly using graph theoretical notations.

\section{Degree:}

In an $\mathrm{ES}(\mathrm{n}, \mathrm{k})$ each PE is directly connected to (n-1) neighbouring PEs of the same Star and to a NC at the next higher level. So the degree of a PE is $n$. The degree of a NC other than the top most NC is $(n !+(n-1)+1)$ or $(n !+n)$

\section{Diameter}

Theorem 1: The diameter of Extended Star ES(n,k) is

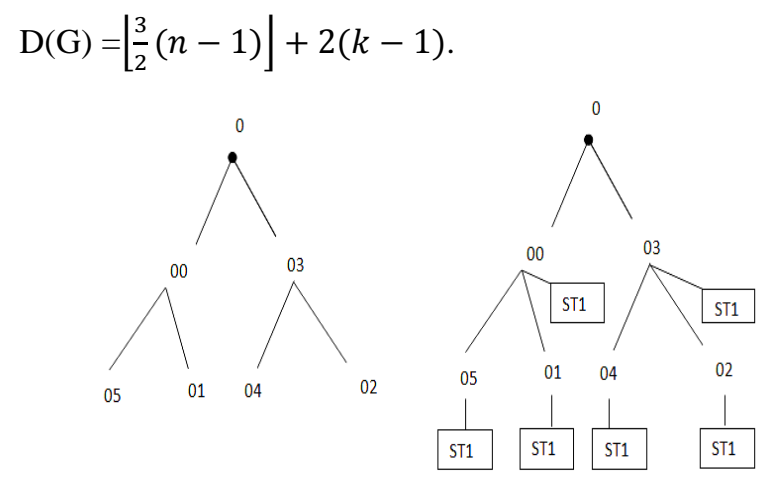

(a)

(b)

Figure $3(a, b)$. Spanning Tree of $\operatorname{ES}(3,1)$ and $\operatorname{ES}(3,2)$

\section{Average Distance:}

For two types of communications: local and global in ES there are two average distances, namely, local and global average distance.

(i) Local Average Distance:

Theorem 2: The local average distance of the $\operatorname{ES}(\mathrm{n}, \mathrm{k})$ is $n-4+\frac{2}{n}+\sum_{i=1}^{n} \frac{1}{i}$.

(ii) Global Average Distance:

Theorem 3: The expression for the global average distance in an $\operatorname{ES}(\mathrm{n}, \mathrm{k})$ is given by $\bar{d}=\frac{\sum d N_{d}}{p+M}$, where, $N_{d}$ is the number of processors at a distance $d$ from the source node. $p$ is the total number of PEs and $\mathrm{M}$ is the total number of NCs.

Nodes:

Theorem 4: The total number of nodes in an ES (n, k) denoted by $p$ is given by $p=n !^{k}+\frac{n !^{k}-1}{n !-1}$

Links:

Theorem 5: The total number of links $\mathrm{E}$ in an $\mathrm{ES}(\mathrm{n}, \mathrm{k})$ is

$$
E=\frac{1}{2}(n+1) ! \times \frac{(n !)^{k}-1}{n !-1}
$$

Cost:

Theorem 6: The cost of the $\mathrm{ES}(\mathrm{n}, \mathrm{k})$ network is given by

$\xi=\left(\left\lfloor\frac{3}{2}(n-1)\right\rfloor+2(k-1)\right) \times \frac{\left.n \times(n !)^{k}+(n !+n) \times\left((n !)^{k}-1\right) /(n !-1)\right)}{\left.(n !)^{k}+(n !+n) \times\left((n !)^{k}-1\right)\right)}$.

\section{Message Traffic Density:}

The performance is very important in handling the message traffic. Assuming that each node is sending a message to a node at a distance $d$, on an average, the message traffic density is defined by, $\rho=\frac{\bar{d} N_{t}}{E}$, where $E$ is the total number of communication links, $N_{t}$, is total 
number of nodes $N t=(p+\mathrm{M})$ and $\bar{d}$ is the average message distance.

Table 1 shows the simulated result for the values of average node distance $\bar{d}, N, M, E$ and $\rho$ for $\operatorname{ES}(3, k)$ for different values of $\mathrm{k}$ keeping $\mathrm{n}=3$.

\section{Extensibility:}

The ES is hierarchical in nature and can be built by extension of the number of levels without affecting the structure of the basic module. The most important advantage of this property is that the degree of any node remains the same, independent of the number of nodes in the network and hence allows for further expansion. The different topological parameters of the proposed Extended star graph are compared with those of the Star graph in Table 2. In the next section the performance analysis of the Extended star network is presented.

TABLE I. Message Traffice Density $(\rho)$ of $\operatorname{ES}(3, \mathrm{k})$

\begin{tabular}{|c|c|c|c|c|c|}
\hline $\boldsymbol{k}$ & $\overline{\boldsymbol{d}}$ & $\boldsymbol{P}$ & $\mathbf{M}$ & $\mathbf{E}$ & $\rho$ \\
\hline 1 & 1.5 & 6 & 1 & 12 & 0.85 \\
\hline 2 & 3.23 & 42 & 7 & 84 & 1.884 \\
\hline 3 & 5.23 & 258 & 43 & 516 & 3.05 \\
\hline 4 & 7.26 & 1554 & 259 & 3108 & 4.22 \\
\hline 5 & 9.29 & 9330 & 1555 & 18660 & 5.33 \\
\hline
\end{tabular}

TABLE II. Comparison of basic parameters of Star and Extended star

\begin{tabular}{|c|c|c|}
\hline Parameter & n-Star & Extended star \\
\hline Size/Nodes & $n !$ & $n !^{k}+\frac{n !^{k}-1}{n !-1}$ \\
\hline Links & $n ! \times\left(\frac{n-1}{2}\right)$ & $\frac{1}{2}(n+1) ! \times \frac{(n !)^{k}-1}{n !-1}$ \\
\hline Node degree & $\mathrm{n}-1$ & $n !+n$ \\
\hline Diameter & $\left\lfloor\frac{3}{2}(n-1)\right\rfloor$ & $\left\lfloor\frac{3}{2}(n-1)\right\rfloor+2(k-1)$ \\
\hline Average distance & $n-4+\frac{2}{n}+\sum_{i=1}^{n} \frac{1}{i}$ & $\bar{d}=\frac{\sum d N_{d}}{p+M}$ \\
\hline Fault Diameter & $\begin{array}{l}\text { If } n=3 \text { or } 4 \text { then original } \\
\text { diameter }+2 \text { And if } n>4 \text { then } \\
\text { original diameter }+1 \text {. }\end{array}$ & $\left\lfloor\frac{3}{2}(n-1)\right\rfloor+2 k-1$ \\
\hline Cost & $(n-1)\left\lfloor\frac{3}{2}(n-1)\right\rfloor$ & $\begin{array}{r}(n !+n)\left[\frac{3}{2}(n-1)\right]+2(k \\
-1)\end{array}$ \\
\hline
\end{tabular}

\section{Performance Analysis of Extended Star}

\section{A. Fault Tolerance}

The ES network is a hierarchical structure of n-star with PEs at lowest level which provides additional path through the network controller there by increasing the fault tolerance property of the network as compared to the star graph. For all the PEs, the node degree is $n$. This means that the network can tolerate up to $(n-1)$ faults.

\section{B. Fault Diameter:}

In an IN, if one or more links (nodes) is faulty, then the network gets deformed resulting in a new diameter, called the Fault diameter $d_{f}$, where $d$ is defined as the maximum diameter of any graph obtained from $G$ by deleting at most $f$ vertices. The fault diameter should be close to the original diameter.

Theorem 7: For $\mathrm{ES}(\mathrm{n}, \mathrm{k})$ the fault diameter is $\mathrm{d}_{f}=$ $\left\lfloor\frac{3}{2}(n-1)\right\rfloor+2 k-1$.

\section{Cost Effectiveness Factor:}

Theorem 8: The cost effectiveness of $\mathrm{ES}(\mathrm{n}, \mathrm{k})$ is derived as $C E F(p)=\frac{1}{1+\rho \times \frac{(n+1) !}{2} \times\left(1-\frac{(n !)^{k}}{p}\right)}$

Proof: In general the number of links is a function of the number of nodes that is $E=f(p)$. The total number of processors links in ES is given by

$$
\begin{aligned}
p=(n !)^{k}+\frac{(n !)^{k}-1}{n !-1}, E=\frac{1}{2} & (n+1) ! \times \frac{(n !)^{k}-1}{n !-1} \\
= & \frac{(n+1) !}{2} \times\left(p-(n !)^{k}\right)=f(p)
\end{aligned}
$$

So, $g(p)=\frac{f(p)}{p}=\frac{\frac{(n+1) !}{2} \times\left(p-(n !)^{k}\right)}{p}=\frac{(n+1) !}{2} \times\left(1-\frac{(n !)^{k}}{p}\right)$

Where $g(p)$ is the ratio of number of links to the number of processors and $\rho$ is the ratio of link cost to processor cost. Hence,

$$
C E F(p)=\frac{1}{1+\rho g(p)}=\frac{1}{1+\rho \times \frac{(n+1) !}{2} \times\left(1-\frac{(n !)^{k}}{p}\right)}
$$




\section{Time Cost Effectiveness Factor:}

Theorem 9: The time cost effectiveness factor of the ES network is given by

$\operatorname{TCEF}(\mathrm{p}, \mathrm{ES})=\frac{1+\sigma}{1+\rho \frac{(n+1) !}{2} \times\left(1-\frac{(n !)^{k}}{p}\right)+\frac{n !-1}{(n !)^{k+1}-1}}$.

Proof: In Theorem 8 , the $g(p)=\frac{(n+1) !}{2} \times\left(1-\frac{(n !)^{k}}{p}\right)$

For any IN topology, the TCEF is given by

$$
\operatorname{TCEF}\left(\mathrm{p}, \mathrm{T}_{\mathrm{p}}\right)=\frac{1+\sigma T_{1}^{\alpha-1}}{1+\rho g(p)+T_{1}^{\alpha-1} \sigma / p}
$$

where $T_{1}$ is the time to solve the problem by uniprocessor system with fastest sequential algorithm and $\mathrm{T}_{\mathrm{p}}$ is the time of parallel algorithm using a multiprocessor system having $p$ processors. Next $\rho=$ of link cost/ processor cost and $\sigma=$ cost of penalty/cost of processors. For a linear time penalty in $\mathrm{T}_{\mathrm{p}}, \alpha=1$.

$p=(n !)^{k}+\frac{(n !)^{k}-1}{n !-1}=\frac{(n !)^{k+1}-1}{n !-1}$, So $\frac{1}{p}=\frac{n !-1}{(n !)^{k+1}-1}$

Then $\operatorname{TCEF}(p, E S)=\frac{1+\sigma}{1+\rho \frac{(n+1) !}{2} \times\left(1-\frac{(n !)^{k}}{p}\right)+\frac{n !-1}{(n !)^{k+1}-1}}$

Hence the result.

\section{Comparison of Isoefficiency for the Proposed Network}

The Isoefficiency determines the ease with which the parallel system can maintain the constant efficiency and hence can achieve scalability. The Isoefficiency $\left(\mathrm{f}_{\mathrm{e}}\right)$ is defined as follows:

Generally the parallel run time $\left(T_{p}\right)$ for ' $p$ ' processing elements and input size ' $w$ ' is defined as [12]

$$
\mathrm{T}_{p}=\frac{w+T_{o}(w, p)}{p}
$$

where $T_{0}$ is the overhead of the system due to idling of the processor. The speedup $(S)$ is defined as

$$
S=\frac{W}{T_{p}}
$$

The Isoefficiency $f_{e}(p)$ is defined as

$$
f_{e}(p)=\frac{s}{p}=\frac{1}{1+T_{o}(w, p) / w}
$$

If a system can maintain constant Isoefficiency for different problem sizes then it is scalable. The value lies between 0 and 1. Using Eq. 3, the Isoefficiency metric for all the networks is evaluated. For evaluation the input size ' $w$ ' is specified and is stated in Table 3.

TABLE III. Problem size specification

\begin{tabular}{|c|c|}
\hline Size(w) & Scale \\
\hline $50-100$ & Small scale \\
\hline $100-500$ & Medium scale \\
\hline $500-1000$ & Big scale \\
\hline $1000-10000$ & Large scale \\
\hline
\end{tabular}

\section{Results and Discussions}

Next the different parameters of the Extended star (ES) network are evaluated and compared with the contemporary networks.

The comparison of the diameter of ES is shown in Fig. 4. The diameter of the ES network has been compared with that of the EH, EVH, Star graph, HC and the Crossed cube. The result reveals that at lower dimensions the ES network is having the lowest value. When increase in dimension the total number of nodes in the ES network increases than that of the parent networks. Hence, the diameter also increase as the system is scaled up. Next in Fig. 5, the cost versus dimension of the $\mathrm{ES}(k, l)$ network is compared with that of the $\mathrm{EH}$, EVH and ECC with fixed $k$ and varying $l$. The result shows that the ES network possesses the lowest value and slower growth rate with higher packing density at same dimension. At lower $n$ the cost of ES is higher than that of ECC as it contains more nodes. The variations of the cost effectiveness and time cost effectiveness factors for the ES network are shown in Fig. 6 and 7 respectively. The TCEF attains its maximum at dimension 12 for ES network with 3-star as the base.

The comparison of the two terminal reliability for ES network is shown in Figs 8 and 9. The Fig. 8 shows that the ES network is highly reliable than the EVH for all $n$. The graph is plotted against the dimension with constant link and processor failure rate at mission time $1000 \mathrm{Hrs}$. Next the reliability of both ES and ECC is compared with respect to mission time. Here the basic building blocks are $\mathrm{CC}_{3}$ and 3-Star. The Extended Star network exhibits better reliability values up to a mission time of 5000Hrs. Beyond that both the networks become equally reliable as shown in Fig. 9. The Isoefficiency graph in Fig. 10

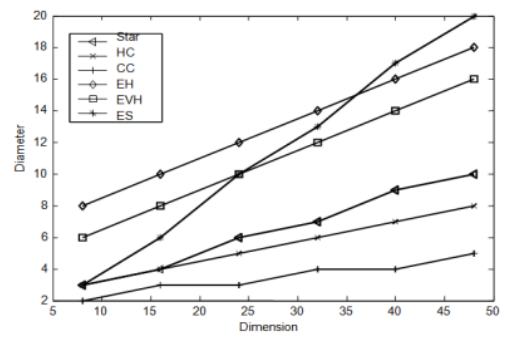

Figure 4. Comparison of diameter of ES network shows the value of the ES network never crosses 1 . The $\mathrm{EH}$ and ECC network posses same number of nodes and thus both graphs completely match. The up and down nature of the graph for ECC and EH network indicates inferior usability. But the ES network exhibits better scalability for small scale networks (50 to 200) and is constantly scalable for medium as well as big scale problems (1000 to 10000).

\section{Conclusion}

The current work discussed about a cost effective and reliable parallel interconnection network called the Extended star. A through approach for constructing the proposed network using the principle of extension is 
discussed. It also presented an in depth analysis on its various topological parameters through illustrations. Due

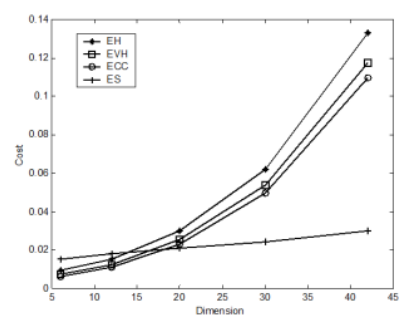

Figure 5. Comparison of cost of ES network

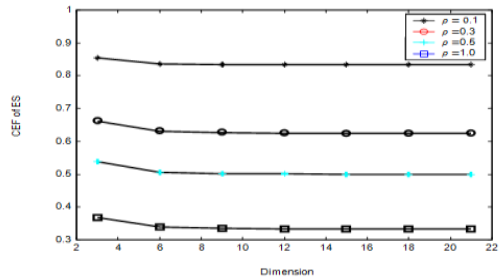

Figure 6. Cost-effectiveness factor of extended star network

to extension the network's performance is improved in terms of fault tolerance and reliability. The ES topology is found to be maximally fault tolerant and scalable. The detailed analysis of results reveals that ES network is better than its parent networks: $\mathrm{EH}, \mathrm{CC}$, Star and the EVH and also ECC network.

\section{References}

[1] L.N. Bhuyan and D. P. Agrawal, "Performance of Multiprocessor Interconnection Network", IEEE Computers, (1989).

[2] T.Feng, “A Survey of Interconnection Networks", IEEE Computers, 1(4),pp.12-27, (1981).

[3] Y Saad and M H. Schultz, "Topological Properties of Hypercube"; IEEE Transactions on Computers, 1988, vol. 37 no. 7: 867-872.

[4] Kemal Efe, "The Crossed Cube Architecture for Parallel Computation"; IEEE Tran. On Parallel and Distributed Systems, Sept 1992, 3(5): 513-524.

[5] Akers S. B. and Krishnamurthy B., "The Fault-tolerance of Star Graphs", Proceedings of International Conference on Supercomputing, pp. 270, (1987)

[6] Ahmed EI-Amawy and Shahram Latifi. "Properties and Performance of Folded Hypercubes", IEEE Transactions on Parallel and Distributed Systems, Jan-1991, 2(1): 31-42.

[7] J M Kumar and L M. Pattnaik, "Extended Hypercube: A Hierarchical Interconnection Network of Hypercubes", IEEE Transactions on Parallel and Disrtibuted Systems, Jan-1992, 3(1): 44-57.

[8] Tripathy C R and Dash R K., "A New Fault-tolerant Interconnection Topology For Parallel Systems" ; IE(I) Journal CP, 2008,89: 8-13.

[9] Yamin Li, Shietung Peng and Wanming Chu , "Metacube: A New Interconnection Network for Large Parallel System", ACSAC02, Australian Computer Science Communications, vol.24(4), pp.29-36, (2001)

[10] N. Adhikari, "Extended Crossed Cube : A New Fault Tolerant Interconnection Network", Int. Journal of Engineering Science Invention, Vol.6, No.9, pp. 60-70, (2017).

[11] N. Adhikari and B. Nag, "On Topological Properties of A Star based Large Scale Parallel System”, Proceedings of ETNCC2011,

International Conference on Emerging Trends in Networks and Computer Communications, IEI Udaipur Section April 22-24, (2011).
[11]

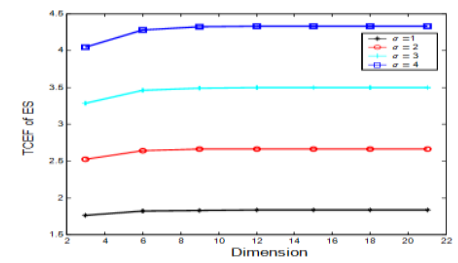

Figure 7. TCEF Vs dimension of Extended star network

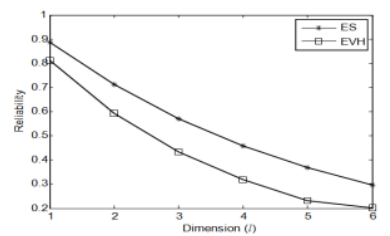

Figure 8. Two terminal reliability evaluation of $\operatorname{ES}(3,1)$ network

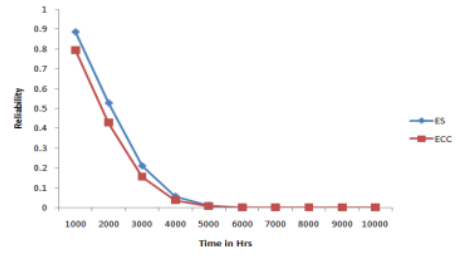

Figure 9. Comparison of reliability of ES and ECC

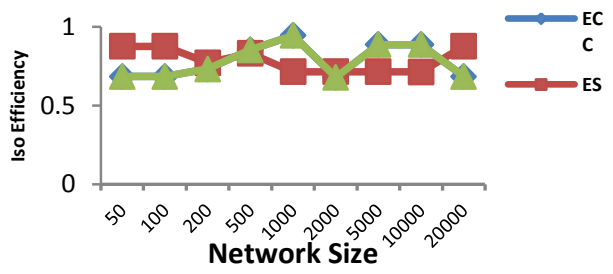

Figure 10. Comparison of Isoefficiency of the networks

[12] Grama A., Gupta A., Karypis G. and Kumar V.: Introduction to Parallel Computing, Pearson Education, 2003.

About Author

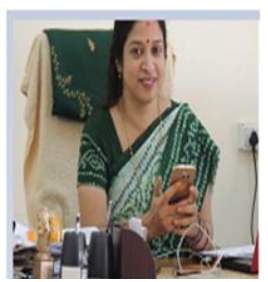

Dr Nibedita Adhikari is working in Biju Patnaik University of Technology, Odisha, India. She has done Maters in Mathematics, MCA from G M College (Autonomous) Sambalpr, India, MTech in Computer Science and Engineering from National Institue of Technology, Rourkela, India and $\mathrm{PhD}$ in Computer Engineering from Sambalpur University. Her area of interests include Computer Architecture, Parallel and Distributed Systems and Soft Computing Techniques. 